\title{
Concomitant direction and distance aftereffects of sustained convergence: A muscle potentiation explanation for eye-specific adaptation
}

\author{
KENNETH R. PAAP and SHELDON M. EBENHOLTZ \\ University of Wisconsin, Madison, Wisconsin 53706
}

\begin{abstract}
Fixating a target for 6 min was shown to produce distance aftereffects that varied in direction and magnitude as a linear function of the convergence angle. Eye-specific direction aftereffects also were obtained in a nasal direction under conditions that produce increased perceived distance and in a temporal direction under conditions that produce decreased perceived distance. These aftereffects were shown to be sensitive to the range of horizontal versional eye movements that accompany the near or far convergence positions maintained during exposure. The results provide a logical alternative to perceptual learning accounts of eye-specific adaptation.
\end{abstract}

Maintaining the eyes in a fixed or restricted range of convergence positions may induce a muscle potentiation aftereffect in the subset of extraocular muscles innervated to achieve and sustain those postures. Muscle potentiation refers to an involuntary component of continued innervation in the direction of previous muscular stimulation. Since the increase in muscle tension is involuntary, registration of eye-position information based on the monitoring of voluntary motor signals should be in error to the degree that the potentiation effect must be overcome. Assuming that the potentiation effect itself is not registered via an inflow from proprioceptive units in the extraocular muscles, it follows that any perceived spatial dimension that requires compensation for or registration of ocular rotation should undergo changes consistent with the felt position of the eyes. Accordingly, changes in perceived distance should occur whenever the eyes have been maintained predominantly in near or far convergence positions. For example, if the medial recti of each eye are potentiated by maintaining the eyes in a near convergence position, then it would be as if each eye was being involuntarily pulled nasally. Therefore, in order to fixate a visual target, the observer would have to overcome the additional innervation due to potentiation by the same amount of voluntary innervation in the opposite direction, i.e., in a temporal direction that would normally result in a smaller convergence angle. This change in

This article is based on a portion of a dissertation submitted to the University of Wisconsin in partial fulfillment of the requirement for the PhD degree (Paap, 1975). This research was supported in part by Grant MH13006 to the second author. Requests for reprints should be sent to Kenneth $R$. Paap, who is now at the Department of Psychology, New Mexico State University, Las Cruces, New Mexico 88003. registered convergence should lead to an increase in the apparent distance to the target. Conversely, maintaining a far convergence position should potentiate the lateral recti, require additional voluntary innervation to the medial recti, and result in a decrease in the apparent distance to the target.

Ebenholtz and Wolfson (1975) have recently reported a series of experiments using a mirror stereoscope to test these predictions across a wide range of symmetrical convergence positions. The first seven experiments show that fixating an inducing stimulus for 6 min produces shifts in the apparent distance to a test target that vary in direction and magnitude as an increasing linear function of the reciprocal of the convergence distance required during the induction interval. As predicted, far convergence distances produce shifts toward a smaller perceived distance, while near convergence distances produce shifts toward a greater perceived distance. The fitted linear function crossed over from a decrease in perceived distance to an increase at a convergence distance of $32.4 \mathrm{~cm}$ (a convergence angle of about $11.4 \mathrm{deg}$ ). This was interpreted as representing the point of balance in aftereffect between the lateral and medial extraocular muscles of each eye, i.e., a physiological position of rest (PPR) for the horizontal vergence system.

If muscle potentiation is the cause of the distance aftereffects reported by Ebenholtz and Wolfson, then there ought to be concomitant aftereffects in egocentric radial direction when direction is judged by each eye alone. If sustained near convergence potentiates the medial recti, then each eye should be reflexively innervated in the nasal direction. Consequently, shifts in the apparent straight-ahead to the left and right would be expected for the right and left eyes, respectively. Far convergence positions that potentiate the lateral recti would, of course, 
result in a complementary pattern of aftereffects. Craske and Crawshaw (1974) obtained concomitant direction and distance aftereffects in a near convergence condition when subjects inspected their feet for $3 \mathrm{~min}$, but no significant effects when control subjects inspected a plain white disk. However, Ebenholtz and Wolfson's (1975) reanalysis of the Craske and Crawshaw distance aftereffects showed no significant differences between experimental and control means and a control mean significantly different from zero, $t(15)=1.91, p<.05$. The present authors' reanalysis of the direction aftereffects also showed no significant differences between experimental and control means, but, in this case, the control mean did not significantly differ from zero. Craske and Crawshaw based their conclusion, that the control group showed no effect, on a two-tailed F test. The marginal effect is to be expected because of the brief inspection interval and also because the observation distance $(26.8 \mathrm{~cm})$ was near the PPR $(32.4 \mathrm{~cm})$ reported by Ebenholtz and Wolfson in Experiments I-VII.

The purpose of the present set of studies was to determine the conditions under which eye-specific aftereffects are likely to occur following simple sustained convergence as compared to those obtained in prism adaptation studies following exposure to informational discrepancy (Foley, 1974; Foley \& Miyanshi, 1969; Hajos, 1968). A favorable comparison would provide additional support for the view that adaptation to wedge prisms results from potentiation of the extraocular muscles and not from perceptual learning (Ebenholtz \& Wolfson, 1975; Paap \& Ebenholtz, 1976).

\section{EXPERIMENT 1}

The goal of the first experiment was to replicate the findings of Ebenholtz and Wolfson (1975) on distance aftereffects using wedge prisms rather than a mirror stereoscope to manipulate the inducing convergence positions. It was necessary to establish the PPR for the stimulus conditions to be used in the following experiments since the empirically determined PPR may vary with changes in the inducing or test stimuli (Ebenholtz \& Wolfson, 1975).

\section{Method}

The observer's head was fixed in a chinrest and brace. A crossing pattern of five light-emitting diodes (FLV-102, red light) located at a viewing distance of $41.3 \mathrm{~cm}$ served as both the inducing and test stimulus. The stimulus was centered in the median plane of the observer's head and at eye level. Center to center, each light was $1 \mathrm{deg}$ of visual angle from any adjacent light. thus, the projected height and width of the stimulus was about 3 deg.

All tests were conducted with normal vision, i.e., the prisms were removed. The distance-test procedure required each observer to fixate the center light of the pattern and to indicate its apparent distance by moving his unseen hand in a slide to a point where the tip of his finger felt to be at the same distance as the visual target, i.e., directly beneath it. The instructions stressed that the observer should only be concerned with how far away the visual target looked and to disregard any nonperceptual factors that might influence his opinion of its true or objective location. The scale running adjacent to the path of the slide permitted its position to be measured to the nearest 10th of a centimeter. Two distance judgments were obtained before and again after a 6-min inducing interval, during which the observer simply fixated the center light of the stimulus with his hands resting in his lap. Starting positions were counterbalanced approximately $10 \mathrm{~cm}$ on either side of the correct position. Between judgments, observers were instructed to close their eyes. Both the test and induction procedures were conducted in the dark and only the stimulus lights were visible to the observer. The posttest-pretest differences were taken as a measure of the aftereffect.

Five groups of seven subjects each were run alternately with wedge prisms that required convergence positions of 31.8 (20 D base-out), 18.1 ( $8 \mathrm{D}$ base-out), 9.0 (no prisms), 4.4 (4 $\mathrm{D}$ base-in), and $-0.1,(8 \mathrm{D}$ base-in) degrees in order to fixate the inducing stimulus. To insure that the observers were maintaining a convergence distance appropriate to their group, they were also instructed to report if the stimulus broke apart into double images. Seven $(31.8 \mathrm{deg}), 2$ (18.1 deg), 0 (9.0 deg), 4 (4.4 deg), and 8 $(-0.1 \mathrm{deg})$ subjects failed to maintain single vision and were replaced in each of the indicated groups.

\section{Results and Discussion}

The aftereffects, from the nearest convergence distance to the furtherest, were $+4.51,+3.00$, $+0.99,-2.24$, and $-2.83 \mathrm{~cm}$. This pattern of aftereffects is consistent with the potentiation hypothesis. Near convergence positions produced shifts in the direction of increased distance estimates $(+)$, while far convergence positions resulted in shifts in the direction of decreased distance estimates $(-)$. An analysis of variance on the aftereffects showed a significant effect of convergence position, $F(4,30)=$ $11.71, \mathrm{p}<.01$.

In Figure 1, the aftereffects are plotted as a function of the convergence angle. This is equivalent to the Ebenholtz and Wolfson (1975) procedure of plotting aftereffects as a function of the reciprocal of the convergence position. A fitted linear function determined by the method of least squares yields a coefficient of determination, $\mathrm{r}^{2}$, of .90 . This indicates that a linear function can account for $90 \%$ of the variance in obtained shifts in apparent distance to the test stimulus. Substitution in the obtained function, $y=.24 x-2.35$, indicates that a convergence angle of about $9.8 \mathrm{deg}$ can be expected to yield no aftereffect at all. This can be interpreted as the PPR for these conditions and corresponds to a convergencedistance of about $38.0 \mathrm{~cm}$. This compares favorably with the $11.4 \mathrm{deg}(32.4 \mathrm{~cm})$ PPR obtained by Ebenholtz and Wolfson (1975).

\section{EXPERIMENT 2}

The purpose of Experiment 2 was to measure direction aftereffects under conditions known to induce large distance aftereffects in the direction of 


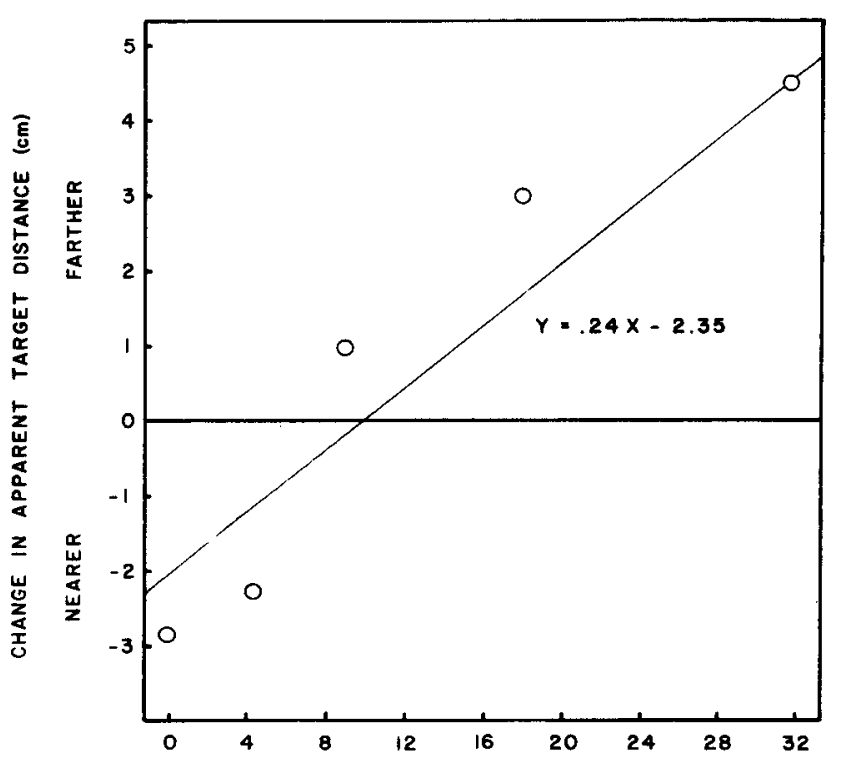

CONVERGENCE ANGLE (dograes)

Figure 1. Distance aftereffects as a function of the convergence angle sustained during the inducing interval.

increased perceived distance in the one case (near convergence) and decreased apparent distance in the other case (far convergence). Accordingly, the conditions that produced maximal effects in the first experiment were used, viz, $31.8 \mathrm{deg}$ (20 D base-out) for a near convergence position and $-0.1 \mathrm{deg}(8 \mathrm{D}$ base-in) for a far convergence position.

\section{Method}

The instructions and apparatus for the distance tests were the same as those used in Experiment 1. Egocentric radial direction was assessed for each eye separately. Prior to each direction judgment, the observer was told to occlude a specific eye with his matching hand. When the observer verbally signaled that his hand was in place, he was instructed to open both eyes (even though he could only view the stimulus array with one), look straight ahead, and fixate that light from an array of lightemitting diodes that appeared to be straight ahead of him. The observer was periodically reminded not to change his mind once he had made his initial choice. The targets were on an equidistant circle with radius of $41.3 \mathrm{~cm}$. All targets were lit when the trial started. The lights were at eye level, $1 \mathrm{deg}$ of visual angle apart, and extended well beyond the limits of the observer's peripheral vision. Lights were sequentially turned off and then on again at a rate of about $2 / \mathrm{sec}$ until the observer verbally indicated that the light he was fixating had been turned off.

The induction procedure and stimulus pattern were the same as that used in Experiment 1, with the exception that the duration of sustained convergence was extended to $10 \mathrm{~min}$. Two binocular distance judgments, two direction judgments with the left eye, and two direction judgments with the right eye were given as practice, followed by an identical set of six judgments that served as the pretest baseline. The order of tests was countutbalanced across observers so that each of the three types of tesits appeared in each of the six serial positions an equal number "of times and was followed by and preceded by each of the other two types of tests an equal number of times. For each observer, the order of pretests and posttests was identical. The mean pasttest-pretest differences were taken as a measure of the aftereffect.

Two groups of 12 subjects each were run alternately in the near and far convergence conditions. The monocular judgments of perceived direction proved to be quite difficult for some observers. Thus, in addition to the previous selection criteria that required single vision throughout the inducing interval, subjects were replaced if their two pretest judgments of direction for either eye exceeded a range of $6 \mathrm{deg}$. Accordingly, 13 subjects were replaced in the near convergence group and 11 in the far convergence group.

\section{Results and Discussion}

The distance aftereffect induced by the near convergence position was $+5.61 \mathrm{~cm}$, and for the far convergence position it was $-3.30 \mathrm{~cm}$. An analysis of variance on the signed difference scores shows that the two means are significantly different from one another, $F(1,22)=32.00, p<.01$. Furthermore, individual $t$ tests show that each shift is significantly different from zero: $t(11)=4.17, p<.01$, for the near convergence group and $t(11)=4.18, p<.01$, for the far convergence group. The distance aftereffects are somewhat larger than those obtained in Experiment 1, but this is not surprising since the induction interval was 4 min longer.

The pattern of direction aftereffects was exactly as predicted. Near convergence positions produced nasal shifts and far convergence positions produced temporal shifts. The mean aftereffect and the number of shifts in the predicted direction for each condition are shown in Table 1. Positive signs indicate a shift to the right, and negative signs a shift to the left. Consequently, the aftereffects at a given convergence position are opposite in sign for the two eyes. For example, the nasal shifts obtained in the near convergence position correspond to a leftward shift in the right eye and a rightward shift in the left eye. This pattern of interaction between eye tested and convergence position was highly significant, $\mathrm{F}(1,22)=16.23, \mathrm{p}<.01$.

When the difference scores are reanalyzed such that positive scores now indicate shifts in the predicted direction rather than to the right, neither the main effect of convergence position, eye tested, nor their interaction achieved significance. This shows that the magnitude of the aftereffects do not significantly differ from one another. However, the grand mean of $1.33 \mathrm{deg}$ was significant, indicating that direction aftereffects were different from zero, $F(1,22)=16.99, \mathrm{p}<.01$.

The direction aftereffects are actually larger than would be expected on the basis of the magnitude of the distance aftereffects. For the 41.3-cm-distant test stimulus, the $5.61-\mathrm{cm}$ increase in apparent distance following sustained near convergence corresponds to a change in half-convergence angle of slightly more than $0.5 \mathrm{deg}$. This is considerably less than the mean direction shift of $1.44 \mathrm{deg}$. Similarly, the $3.30-\mathrm{cm}$ decrease in apparent distance following sustained 
Table 1

Direction Aftereffects in Experiments 2 and 3 (Degrees)

\begin{tabular}{|c|c|c|c|c|c|c|c|c|c|c|}
\hline \multirow{2}{*}{ Eye Tested } & \multicolumn{4}{|c|}{ Experiment 2} & \multicolumn{6}{|c|}{ Experiment 3} \\
\hline & \multicolumn{2}{|c|}{ Near } & \multicolumn{2}{|c|}{ Far } & \multicolumn{2}{|c|}{ Static } & \multicolumn{2}{|c|}{ Narrow } & \multicolumn{2}{|c|}{ Wide } \\
\hline Mean Aftereffect* & -1.21 & +1.08 & +.94 & -1.50 & -1.60 & +1.75 & -1.00 & +1.67 & -.44 & -.29 \\
\hline $\begin{array}{l}\text { Number Shifting in } \\
\text { Predicted Direction** }\end{array}$ & 11 & 10 & 9 & 10 & 8 & 9 & 9 & 9 & 6 & 6 \\
\hline
\end{tabular}

far convergence corresponds to a change in halfconvergence angle of slightly less than $0.5 \mathrm{deg}$ compared to the obtained mean direction shift of 1.22 deg.

In summary, eye-specific direction aftereffects were obtained consistent with potentiation in the medial recti following near convergence and in the lateral recti following far convergence. The magnitude of the change in egocentric radial direction was somewhat larger than would be expected from the obtained changes in perceived distance.

\section{EXPERIMENT 3}

Ebenholtz and Wolfson (1975, Experiments VIII, IX) showed that distance aftereffects can occur under conditions of varied fixation, given that the eyes were maintained predominantly in either near or far convergence positions. However, Foley (1974) failed to find direction aftereffects following sustained near convergence induced by reading a text placed at a viewing distance of 10 in. for as long as $20 \mathrm{~min}$. Therefore, the purpose of the third experiment was to examine the buildup of muscle potentiation under various degree of lateral changes in fixation. Specifically, it was predicted that a narrow range of horizontal eye movements would produce significant distance and direction aftereffects. It was further expected that a wide range of horizontal eye movements would prevent the buildup of any significant effects. The main reason for these predictions stems from the fact that the muscle aftereffects diminishes after the previously innervated muscle has been relaxed or inhibited (Paap \& Ebenholtz, 1976). A small range of eye movements will tend to maintain tension in the yoked extraocular muscles, whereas a large left-to-right shift will cause the agonists to relax more completely than otherwise. The conditions associated with these events will be described more fully below.

\section{Method}

The instructions and apparatus for both the distance and direction tests were the same as those employed in Experiment 2. All observers wore 12-D base-out prisms during the 10 -min induction interval. Three groups of 12 subjects each were run alternately under three conditions of fixation. The static group maintained a steady fixation on the same straight-ahead inducing stimulus that was used in the first two experiments. The prisms required a convergence position of $22.7 \mathrm{deg}$. The two movement groups alternately fixated the center of three different inducing stimuli, identical in configuration to the standard inducing stimulus. For the narrow-range movement group, the center lights on the two peripheral stimuli were symmetrically located 5 deg on either side of the center stimulus. For the wide-range movement group the center lights of the peripheral stimuli were $10 \mathrm{deg}$ on either side of the center stimulus. The observers were verbally instructed to shift their fixation from left to right and back again throughout the induction interval. The peripheral targets were fixated for $8 \mathrm{sec}$ at a time and the center target for $4 \mathrm{sec}$. Thus, each of the three stimuli were fixated for the same amount of total time. The same selection criteria as applied in Experiment 2 resulted in three subjects' being replaced in the static group, five in the narrow-range group, and six in the wide-range group.

\section{Results and Discussion}

The distance aftereffects for the static, narrowrange, and wide-range groups, respectively, were $+4.10,+2.27$, and $-0.48 \mathrm{~cm}$. An analysis of variance on the aftereffects showed a significant effect of fixation condition, $F(2,33)=5.48$, $p<.01$. Since the question of interest is whether or not varied fixation will produce aftereffects comparable to constant fixation, the static group is essentially a control to which the movement groups can be compared using Dunnett's test. The wide-range group shifted significantly less than the static group, $d(33)=3.29, p<.01$, but the narrow-range group did not, $\mathrm{d}(33)=1.67, \mathrm{p}>.05$. Individual $t$ tests on each group's scores are consistent with this analysis, indicating that only the static and narrowrange aftereffects differ significantly from zero, $\mathrm{t}(11)=3.91, \mathrm{p}<.01$, and $\mathrm{t}(11)=4.08, \mathrm{p}<.01$, respectively. These analyses of the distance aftereffects suggest that a narrow range of horizontal eye movements will not effect the induction of muscle potentiation in the medial recti, but that a wider range of movements will prevent the buildup of any effect.

The direction aftereffects for the three fixation conditions are shown at the right in Table 1. A positive sign indicates a shift to the right and a negative sign a shift to the left. An analysis of variance on these aftereffects yielded a significant interaction 
of Eye Tested by Fixation Condition, $F(2,33)=$ $4.12, \mathrm{p}<.01$. This reflects the fact that the differences between the right and left eyes decrease from the static to narrow-range to wide-range groups. Subsequent $t$ tests for the right vs. left eye in each of the three groups support this description, since the left-right comparison was significant only in the first two groups, $t(11)=4.11, p<.01$, for the static group and $t(11)=3.79, p<.01$, for the narrowrange group. When significantly different direction aftereffects are obtained, the apparent straight-ahead shifts to the left in the right eye and to the right in the left eye, just as predicted by the assumption that near convergence positions will induce potentiation in the medial recti.

When the difference scores are reanalyzed such that positive scores now indicate shifts in the predicted direction, rather than to the right, only the main effect of fixation condition was significant, $F(2,33)$ $=5.12, \mathrm{p}<.01$. This indicates that the magnitude of the direction aftereffects depends upon the fixation condition during exposure. Subsequent individual $t$ tests indicate that the mean direction aftereffect for the static group, $+1.68 \mathrm{deg}$, and that of the narrow-range group, $+1.37 \mathrm{deg}$, both differ significantly from zero, $t(11)=2.28, p<.05$, for the former and $t(11)=4.94, p<.01$, for the latter. The +0.07-deg mean for the wide-range group was not significant. Dunnett's test indicates that only the wide-range group aftereffects differed significantly from the static control group, $d(33)=2.14$, $p<.05$. In summary, the analyses of the direction aftereffects are completely consistent with the analyses of the distance aftereffects. Both tests support the hypothesis that a limited range of horizontal eye movements will induce potentiation effects similar to a constant convergence position, but that wider sweeps will not induce significant effects.

Experiment 1 can be interpreted as showing a PPR for the present experimental conditions corresponding to a convergence distance of $38 \mathrm{~cm}$ or a convergence angle of $9.8 \mathrm{deg}$. This suggests that direction aftereffects in the right eye should cross over at inducing eye turns of $4.9 \mathrm{deg}$ to the left and in the left eye with an equivalent rotation to the right. Although the three inducing stimuli all require approximately the same convergence angle $(13.7 \mathrm{deg})$, the ocular deviation of each eye relative to the median plane of the head $(a)$ undergoes considerable variation in the two movement groups. Figure 2 depicts the geometric solution of $\alpha$ for a fixation target placed $x$ degrees to the left of the median plane. Sides $b$ and $c$ of the triangle formed by the center of the pupil, the midpoint of the interocular axis, and the peripheral inducing stimulus are constant throughout the experiment. The length of Side a, given some particular location of the peripheral inducing stimulus, is computed in Step 1 following the law of cosines. Angle $\mathrm{Y}$ is computed in Step 2 for the law of sines. The ocular deviation of each eye relative to the median plane of the head $(\alpha)$ is determined in Step 3 by taking the complement of angle $Y$.

Consider the narrow-range group that inspects inducing stimuli 5 deg to the right and left of straight ahead $(x= \pm 5 \mathrm{deg})$. Fixating either peripheral target requires the nearer eye to adduct $7.3 \mathrm{deg}$ and the eye farthest from the target to adduct $16.1 \mathrm{deg}$. Consequently, fixation angles $7.3,11.3$, and $16.1 \mathrm{deg}$ to the median plane are alternately maintained in each eye throughout the inducing interval. Assuming a PPR at $4.9 \mathrm{deg}$ of adduction, the medial recti of the narrow-range group are never subject to stretch beyond the neutral point. It follows, therefore, that apparent distance should increase and that apparent direction should shift in the direction of medial rectus stimulation. These are precisely the effects obtained.

The wide-range group fixated inducing stimuli $10 \mathrm{deg}$ to the right and left of straight-ahead $(x=$ $\pm 10 \mathrm{deg})$. This larger displacement of the fixation target required the eye nearest the fixation target to adduct only $2.6 \mathrm{deg}$ and the eye farthest from the target to adduct $20.1 \mathrm{deg}$. Thus, each eye, during its excursion from the far left target to the far right, would cross the 4.9-deg PPR. The absence of distance or direction aftereffects with these targets is consistent with the notion that stretching a muscle in the opposite direction of the inducing stimulation will erase or inhibit the buildup of muscle potentiation (Paap \& Ebenholtz, 1976).

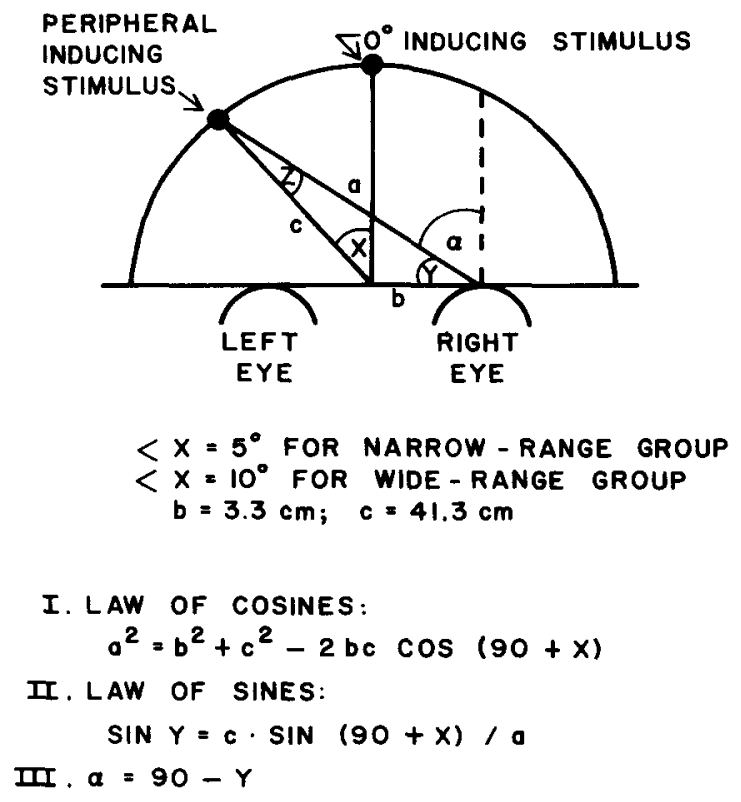

Figure 2. The ocular deviation of the right eye from the median plane of the head $(\alpha)$ in relation to the inducing stimulus displacement $(X)$ from the straight ahead. 


\section{IMPLICATIONS FOR PRISM ADAPTATION}

Current explanations for adaptation to optical transformations hold that visual aftereffects result from perceptual learning. Held (1961), in an expansion of von Holst's work, has developed a version of perceptual learning theory that is based on reafferent stimulation. Reafference is visual input correlated with active voluntary movement on the part of the observer. The necessary condition for adaptation in this theory is a discrepancy between the current relationship between the efferent and reafferent signal and the stored relationship buildup over time from past experience. More recently, Wallach and Frey (1972a, b) and Epstein (1975) have advanced a more general perceptual learning theory termed recalibration. Although they have been working with different types of optical transformations, both investigators agree that a necessary condition for adaptation is informational discrepancy during the exposure interval. There are usually several redundant cues to a given spatial dimension. Some of these cues are within the visual modality, while others may involve proprioception. The introduction of prisms or lenses in a normal visual environment will usually serve to modify one cue system while preserving the veridicality of at least one remaining cue system. The recalibration hypothesis predicts that the dominant or attended source of information will serve to recalibrate the secondary cue system.

A critical, and almost always overlooked, aspect of the majority of adaptation studies is that the optical devices together with the task demands may serve to bias the observer's eye position during the exposure interval. Typically, the pattern of postural bias exactly matches the pattern of inducing stimulation required for muscle potentiation to predict the perceptual changes usually attributed to recalibration. A muscle potentiation interpretation of adaptation to lateral displacement has been previously presented (Paap \& Ebenholtz, 1976). The results of the present set of experiments permit a similar analysis of the changes in perceived distance and monocular direction that usually follow from exposure to base-out or base-in prisms. The extension of the potentiation explanation to two more types of adaptation is important since the theories are quite distinct in the implications for plasticity within the visual system. Potentiation implies nothing more than a peripheral adjustment to unusual loads on the extraocular muscles, whereas recalibration postulates a central learning process that should be capable of accommodating a much wider range and variety of optical rearrangements.

\section{Distance Adaptation}

In a series of experiments, Wallach and his associates (Wallach \& Frey, 1972a, b; Wallach, Frey, \& Bode,
1972) had observers wear "near" glasses equipped with base-out prisms that, for any given fixation distance, forced the eyes to converge 5.7 deg nearer than normal and -1.5-D lenses that produced an increase in accommodation corresponding to the convergence change caused by the prisms. Occassionally, "far" glasses were also used. These are equipped with 2.5-D base-in prisms and +0.75-D lenses that force the eyes to rotate towad a more relaxed convergence position by $2.85 \mathrm{deg}$. A constant factor across all the various exposure conditions tested was that there was an opportunity for the relationship between the modified oculomotor cues (convergence and accommodation) and perceived distance to be recalibrated by another distance cue that remained veridical during the exposure period. Exposure durations of 20 or $30 \mathrm{~min}$ consistently produced shifts in perceived size, depth, and distance that supported the view that the oculomotor cues to distance had been recalibrated so that objects viewed through the glasses would tend to be seen at more veridical distances. That is, the near glasses that required convergence distances closer than the actual distance adapted in the direction of increased distance, whereas the far glasses that required convergence distances further than the actual distances adapted in the direction of decreased distance.

If the near glasses restrict the range of convergence positions to the extent that tension is sustained in the medial recti, then similar conditions of extraocular muscle-stimulation exist in both Wallach's procedure and our own. Consequently, at least some component of the adaptive shifts in Wallach's experiments could be due to potentiation. In order to support this line of reasoning, it is necessary to show that the induction procedures used in the present studies are free of any informational discrepancy that could serve to recalibrate the convergence cue to distance and that aftereffects can occur with a moving eye as well as under stable fixation.

Accommodation could have served to recalibrate the fusional-convergence cue in the present investigation. Since accommodation distance was fixed at $41.3 \mathrm{~cm}$, no conflict, and therefore no aftereffects, should occur when the inducing stimulus is at that convergence distance. Since this value is very near the $38.0 \mathrm{~cm}$ PPR obtained in Experiment 1, recalibration could predict the obtained pattern of distance aftereffects. However, it is not clear how a recalibration theory could account for the concurrent direction aftereffects obtained in Experiments 2 and 3. Furthermore, the recalibration hypothesis clearly fails to predict the distance aftereffects in Experiments VIII and IX of Ebenholtz and Wolfson (1975). Here, convergence was manipulated by physically varying the location of the inducing stimuli rather than by means of a mirror stereoscope or wedge prisms. Since no optical devices were used, all 
distance cues were potentially veridical and there simply was no informational discrepancy. This fact, coupled with the similarity in obtained functions, suggests that all of the shifts reported by Ebenholtz and Wolfson and in the present study are due to potentiation and not to recalibration.

The second requirement in generalizing the potentiation studies to Wallach's adaptation studies, that the aftereffects can occur with a moving eye as well as under constant fixation, is now well documented. Neither a constant convergence distance (Ebenholtz \& Wolfson, 1975) nor direction (Experiment 3 of the present study) is required in order to produce distance aftereffects. Experiment 3 does show that the range of versional eye movements must be limited to $10 \mathrm{deg}$ if equivalent shifts are to be obtained and to less than $20 \mathrm{deg}$ if significant shifts are to be obtained. This range does not seem unreasonable for the typical prism exposure condition, since any target requiring a large ocular deviation is probably inspected with a combination of head and eye rotation.

\section{Eye-Specific Adaptation}

Convergence position was manipulated in the present report by varying the magnitude and orientation of wedge prisms mounted with their bases opposing one another. Furthermore, the convergence position was controlled by having the observer report if the inducing stimulus broke apart into double images. If the observer could not easily recover fusion or if his reports of double images were frequent, he was dismissed and subsequently replaced. This selection procedure was enforced to insure that the eyes were actually rotated in equal and opposite directions, and therefore that the stimulus for potentiation was the same for the medial (near convergence positions) or lateral (far convergence positions) recti of each eye. The results clearly indicated nasal shifts in each eye following potentiation of the medial recti and temporal shifts in each eye following potentiation of the lateral recti.

This pattern of direction aftereffects may be referred to as eye-specific adaptation (Ebenholtz, 1970), indicating that the apparent direction of a visual target has changed in one direction when tested with the right eye and in the opposite direction when tested with the left eye. Therefore, from the muscle potentiation point of view, eye-specific adaptation would be expected whenever the magnitude of the prisms permits the observer to maintain fusion during the exposure interval. However, large disparities will exceed the capabilities of the oculomotor system to rotate so that corresponding retinal points will intercept similar patterns in the optic array. When fusion is not possible, eye posture, and hence the direction of potentiation aftereffects, is difficult to predict. If both eyes move identically, as de- manded by Hering's law of equal innervation, then the inducing posture for both eyes would be the same throughout the exposure interval. This condition would produce adaptive shifts for the one eye and counteradaptive shifts, relative to the prism orientation, in the other eye. Alternately, large disparities which do not permit fusion may still be adequate stimuli to induce an incomplete fusional-vergence movement. In this case, the postural bias induced would be less than that obtained with complete fusion. Accordingly, weak eye-specific aftereffects may be obtained for some observers. Finally, some observers may oscillate between these two tendencies. Their aftereffects would be some balance between the relative effects of Hering's law and the fusion reflex.

The reported findings are in line with the preceding analysis. Small disparities, especially in the more flexible horizontal system have produced eye-specific changes consistent with those reported in Experiments 2 and 3. Hajos (1968) had observers wear 10.5-D base-out prisms for a period of 9 days. Apparent direction was tested by having the observer point with his unseen hand at a monocularly presented target. The final level of adaptation was about $2.8 \mathrm{deg}$ in the opposite direction in each eye. Eyespecific effects as large as $4 \mathrm{deg}$ were reported in a similar study by Foley (1974) with 10-D prisms mounted either base-in or base-out. These shifts are larger than the 1- to 2-deg shifts obtained in the present study, but the former were obtained with exposure durations of at least $2.5 \mathrm{~h}$ compared to the $8 \mathrm{~min}$ of induction used in the latter. At $15 \mathrm{~min}$ of exposure, the time of the first posttest, Foley's effects average less than 2 deg with base-out prisms and only about $0.5 \mathrm{deg}$ with base-in prisms. Thus, aftereffect magnitude does seem reasonably comparable in the two procedures.

The vast majority of the observers in Foley's other conditions (20 D base-out, $20 \mathrm{D}$ base-in, and $10 \mathrm{D}$ base-up and down) showed aftereffects which were controlled in direction by one eye. A similar pattern of results was reported in an earlier study by Foley and Miyanshi (1969). Here, significant adaptive shifts were obtained for only the left eye following exposure to a 20-D base-up prism over the right eye and a 20-D base-down prism over the left eye. Since all of these conditions exceed the capability of observers to achieve fusion, the results are consistent with the muscle potentiation hypothesis.

Foley (1974) also views the presence or absence of fusion as critical, but for other reasons. She argues that double vision forces the observer to select or attend to only one or the other of the two fields. The chosen feedback channel controls the aftereffect in a reafference based (Held, 1961) version of perceptual learning theory. Foley rejected an alternative explanation based on sustained convergence for the 
adaptive aftereffects, since control observers, who were simply exposed to reading an essay at a viewing distance of $10 \mathrm{in}$. , failed to give either consistent direction or distance aftereffects. The choice of a control task was probably a poor one, since it is likely that the reading required a wider range of horizontal versional eye movements than normally required by walking in hallways or similar prism exposure activities. Assuming an interocular distance of $6.6 \mathrm{~cm}$, fixating the center of the page of text required a convergence angle of $14.8 \mathrm{deg}$. Therefore, assuming a PPR convergence angle of $9.8 \mathrm{deg}$, sustained convergence in this position should produce both distance and direction aftereffects. However, the observers were reading a text that subtended about $19 \mathrm{deg}$ in the horizontal dimension. Following the geometry outlined in Figure 2, fixating the horizontal boundaries of the text requires a maximum abduction of about $5 \mathrm{deg}$. For example, when the observer shifts fixation from the far left to the far right side of the page his right eye turns from a 14-deg rotation to the left to a 5-deg rotation to the right. Assuming a PPR at $4.9 \mathrm{deg}$ of adduction (from the $9.8 \mathrm{deg}$ PPR associated with the symmetrical convergence positions of Experiment 1), each eye during its excursion from the left side to the right side would cross the PPR. The wide-range group of Experiment 3 demonstrates that this is not a sufficient condition for inducing muscle potentiation effects. The conclusion may be drawn that ocular muscle aftereffects are sufficient to explain much of what is now attributed to recalibration or visual change.

\section{REFERENCES}

Enenholtz, S. M. On the relation between interocular transfer of adaptation and Hering's law of equal innervation. Psychological Review, 1970, 77, 343-347.

Ebenholtz, S. M., \& Wolfson, D. M. Perceptual aftereffects of sustained convergence. Perception \& Psychophysics, 1975, 17, 485-491.

EPSTEIN, W. Recalibration by pairing: A process of perceptual learning. Perception, 1975, 4, 59-72.

Craske, B., \& Crawshaw, M. Adaptive changes of opposite sign in the oculomotor systems of the two eyes. Quarterly Journal of Experimental Psychology, 1974, 26, 106-113.

FOLEY, J. E. Factors governing interocular transfer of prism adaptation. Psychological Review, 1974, 81, 183-186.

FOLEY, J. E., \& MIYANSHI, K. Interocular effects in prism adaptation. Science, 1969, 165, 311-312.

Hajos, A. Senumotorische Koordinationsprozesse bei Richtungs Lokalisation. Zeitschrift für Experimentelle und Angewandte Psychologie, 1968, 15, 435-461.

HeLD, R. Exposure history as a factor in maintaining stability of perception and coordination. Journal of Nervous and Mental disease, 1961, 132, 27-32.

PaAp, K. R., \& Ebenholtz, S. M. Perceptual consequences of potentiation in the extraocular muscles: An alternative explanation for adaptation to wedge prisms. Journal of Experimental Psychology: Human Perception and Performance, 1976, 2, $457-468$.

Wallach, H., \& Frey, K. J. On counteradaptation. Perception \& Psychophysics, 1972, 11, 161-165. (a)

WALLACH, H., \& FreY, K. J. Adaptation in distance perception based on oculomotor cues. Perception \& Psychophysics, 1972, 11, 77-83. (b)

Wallach, H., Frey, K. J., \& Bode, K. A. The nature of adaptation in distance perception based on oculomotor cues. Perception \& Psychophysics, 1972, 11, 110-116.

(Received for publication August 16, 1976; revision accepted December $10,1976$. 